\title{
Review of: "CTCF knockout in zebrafish induces alterations in regulatory landscapes and developmental gene expression"
}

\author{
Kenta Nakai ${ }^{1}$
}

1 Tokyo University

Potential competing interests: The author(s) declared that no potential competing interests exist.

In this manuscript, the authors present a variety of experiments to characterize how the CTCF gene can affect the developmental process in zebrafish embryos. Compared with the analogous knockout experiments in mice, the zebrafish embryo can sustain its life to a relatively late stage, probably due to the sake of its maternal gene product. Although there has been controversial on the role of CTCF in controlling gene expression through maintaining and modulating the precise 3D chromatin structure, the authors present rather clear data that the knockdown of CTCF causes significant losses of the 3D chromatin structure, which affect the expression of a number of development-related genes, while a subset of such genes are also affected through another function of CTCF as a transcriptional regulator. Unfortunately, its main conclusions can be mostly derived from the first half of experiments while the latter, also quite extensive, works seem to have failed to draw novel insights in our understanding of 3D chromatin-based developmental control. Nevertheless, the presented data will be a valuable resource for those who are interested in the field. My impression was that the regulation of developmental genes through the change of chromatin landscape is quite subtle and complicated. How such subtle regulations are ensured is still a big mystery. To a suggestion to the authors, I wonder if the abstract could be more informative, reflecting the insights from their latter half of experiments. 